\title{
DNA encapsulation by biocompatible catanionic vesicles ${ }^{\text {ts }}$
}

\author{
Mónica Rosa $^{\mathrm{a}, \mathrm{b}, *}$, Maria da Graça Miguel ${ }^{\mathrm{a}}$, Björn Lindman ${ }^{\mathrm{a}, \mathrm{b}}$ \\ a Chemistry Department, Coimbra University, 3004-535 Coimbra, Portugal \\ b Physical Chemistry 1, Lund University, P.O. Box 124, 22100 Lund, Sweden
}

Received 16 May 2006; accepted 29 July 2006

Available online 9 August 2006

\begin{abstract}
The encapsulation of DNA by catanionic vesicles has been investigated; the vesicles are composed of one cationic surfactant, in excess, and one anionic. Since cationic systems are often toxic, we introduced a novel divalent cationic amino-acid-based amphiphile, which may enhance transfection and appears to be nontoxic, in our catanionic vesicle mixtures. The cationic amphiphile is arginine- $N$-lauroyl amide dihydrochloride (ALA), while the anionic one is sodium cetylsulfate (SCS). Vesicles formed spontaneously in aqueous mixtures of the two surfactants and were characterized with respect to internal structure and size by cryogenic transmission electron microscopy (cryo-TEM); the vesicles are markedly polydisperse. The results are compared with a study of an analogous system based on a short-chained anionic surfactant, sodium octylsulfate (SOS). Addition of DNA to catanionic vesicles resulted in associative phase separation at very low DNA concentrations; there is a separation into a precipitate and a supernatant solution; the latter is first bluish but becomes clearer as more DNA is added. From studies using cryo-TEM and small angle X-ray scattering (SAXS) it is demonstrated that there is a lamellar structure with DNA arranged between the amphiphile bilayers. Comparing the SOS containing DNA-vesicle complexes with the SCS ones, an increase in the repeat distance is perceived for SCS. Regarding the phase-separating DNA-amphiphile particles, cryo-TEM demonstrates a large and nonmonotonic variation of particle size as the DNA-amphiphile ratio is varied, with the largest particles obtained in the vicinity of overall charge neutrality. No major differences in phase behavior were noticed for the systems here presented as compared with those based on classical cationic surfactants. However, the prospect of using these systems in real biological applications offers a great advantage.
\end{abstract}

(c) 2006 Elsevier Inc. All rights reserved.

Keywords: Catanionic vesicles; DNA

\section{Introduction}

The research activity involving gene therapy with either viral or synthetic vectors is extensive and unique. Synthetic vectors have generally been considered to be less efficient than their viral counterparts but have clear advantages [1,2]: ease and variability of preparation; lack of immune response; and unlimited DNA-carrying capacity (allows delivery of human artificial chromosomes [3]). Recent problems in clinical trials with viral vectors, namely a fatality induced by a severe inflammatory

\footnotetext{
is This work is submitted to honor the memory of the great chemist Professor Kunieda, with whom the senior authors had many fruitful contacts and collaborations during a long sequence of years.

* Corresponding author.

E-mail address: acinom@ci.uc.pt (M. Rosa).
}

response [4] and insertional mutagenesis caused by retroviral vectors [5], have further encouraged the study and development of new efficient nonviral vectors. Furthermore, a recent study on nonviral systems suggested that the anti-tumor activity of some cationic entities together with their transfection capability can be explored to successfully improve gene therapy [6]. This study by Dufes et al. [6] could potentially revitalize the advancement of nonviral vectors not only with the purpose of improving the cellular delivery of genetic material, but also to exploit the vector's intrinsic biological activities for additive or synergistic therapeutic effects.

Usually, vesicular transport systems are the vehicles of choice within nonviral gene therapy, liposome-based systems being the most popular ones so far [7]. Due to the growing need for novel engineered vehicles, we present in this study catanionic vesicle systems as DNA carriers. By catanionic systems 
are understood mixtures of cationic and anionic amphiphiles. A number of catanionic amphiphile mixtures have previously been presented in the literature; vesicles were generally found to form spontaneously [8-12], featuring an ease of preparation needed for real applications. Moreover, studies on the interaction between DNA and catanionic vesicles have been presented in the literature [13-16], demonstrating a strong associative phase separation with precipitate formation [14-16], the driving force for this strong association being counterion release [17]. A self-assembly of the DNA-catanionic vesicle complexes into distinct lamellar nanostructures, with encapsulation of DNA within the amphiphile lamellar stacks, was found [14-16]. A highlight of these DNA-catanionic vesicle complexes of great interest for gene delivery applications is the high degree of DNA encapsulation; the underlying mechanism enabling further DNA uptake by the complexes is the release of the anionic entity from the amphiphile bilayer [16].

Another significant reason why nonviral vectors are not successful in the clinic, apart from the inefficient delivery of nucleic acids, is their toxicological effects, which are in particular due to the cationic entities. These toxicity consequences can be avoided by designing new cationic amphiphiles based on novel biocompatible molecules. By incorporating an amino-acid-based engineered cationic amphiphile (arginine$N$-lauroyl amide dihydrochloride, ALA) into our mixtures, they will be much better tolerated by biological systems than conventional cationic amphiphiles. Indeed, cell viability tests performed prove this to be right; cytotoxicity studies for ALA alone and for catanionic mixtures used in this work resulted in cell viability percentages above $90 \%$ (results to be published). ALA has also been reported as being biodegradable [18]. Furthermore, multivalent lipids have been described as superior to their monovalent counterparts as transfection agents [19,20], and ALA is a divalent amphiphile.

In this work we have investigated the interaction of DNA with a mixed system composed of ALA and sodium cetylsulfate (SCS). From an analysis of the supernatant we get a picture of the colloidal behavior and stability. The concentrated phase is investigated with respect to internal structure by SAXS and cryo-TEM, the latter technique also providing information on particle size and shape. The manipulation of the morphology and size of the particles formed by tuning the electrostatics of the system is also explored.

The study complements our previous study of another analogous system with an anionic surfactant of much shorter chain length, sodium octylsulfate (SOS) [16]. It is known that vesicle stability is much dependent on the relative amphiphilicity of the two surfactants [8-10,21]. In addition to a comparison with our previous study on the SOS system, we also include in this report additional data for this system.

\section{Experimental}

\subsection{Materials}

Sodium octyl- and cetylsulfates (SOS and SCS) were obtained from Merck and used as received; arginine- $N$-lauroyl

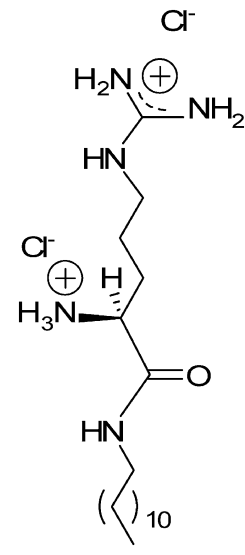

Fig. 1. Chemical structure of the amino-acid-based amphiphile (ALA) used in this study.

amide dihydrochloride (ALA) is a divalent cationic amphiphile (Fig. 1); its synthesis and physiochemical properties can be found in the literature $[18,22,23]$. Double stranded DNA (dsDNA) type XIV from herring testes was purchased from Sigma and used as received. DNA was further investigated by gel electrophoresis to determine its molecular weight; it was found to be polydisperse and have between 400 and 1000 base pairs (bp), with a center of the distribution at ca. $700 \mathrm{bp}$. All DNA concentrations were determined spectrophotometrically considering the molar extinction coefficient of DNA bases to be equal to $6600 \mathrm{M}^{-1} \mathrm{~cm}^{-1}$ [24]. The ratio between the measured absorbances at 260 and $280 \mathrm{~nm}$ of DNA stock solutions was found to be between 1.8 and 1.9, which suggested the absence of proteins [25]. All concentrations are presented per charge. Water was purified using a Millipore Q system.

\subsection{Sample preparation}

Solutions of catanionic vesicles were prepared by making stock solutions of individual amphiphiles, combining them, at the desired composition, to a required amount of water (W) and mixing all components by simple hand agitation. The vesicle solutions used were ALA/SCS/W and ALA/SOS/W. The ALA/SCS solution had a total amphiphile concentration of 0.5 wt\% with a molar charge ratio of ALA to SCS of 2.9. The ALA/SOS one had a total amphiphile concentration of $1 \mathrm{wt} \%$ with a molar charge ratio of ALA to SOS of 1.7. After solution preparation, solutions were left to equilibrate at room temperature for a few days.

Stock solutions of double stranded DNA were prepared at $1 \mathrm{mM} \mathrm{NaBr}$ and added to the vesicle solutions to achieve the required final concentration.

Mixed solutions of DNA and vesicles were prepared at different DNA to vesicle effective net charge ratios, $R=$ $[\mathrm{DNA}] /\left[\mathrm{S}^{+}\right]_{\text {eff }}[26]$, where $\left[\mathrm{S}^{+}\right]_{\text {eff }}$ is the vesicle effective cationic net charge concentration, which is the difference between the concentration of ALA and the anionic amphiphile concentrations (concentrations determined per charge). The next step consisted in centrifuging all samples for $10 \mathrm{~min}$ at $4000 \mathrm{rpm}$. The supernatant was taken off by pipetting the upper part of the centrifuged samples. 


\subsection{Cryogenic transmission electron microscopy}

Cryo-TEM is a powerful technique for direct visualization of colloidal aggregates in aqueous media, provided particle sizes range from 5-10 $\mathrm{nm}$ to $1 \mu \mathrm{m}$. Controlled sample preparation conditions are required: a controlled-environment vitrification system (CEVS), at controlled temperature (to prevent temperature changes) and humidity (to minimize water loss), was used [27]. Vitrified samples were prepared and imaged according to a procedure described in the literature [27-29]. A Philips CM 120 Bio-Twin microscope equipped with a post-column energy filter, using an Oxford CT3500 cryoholder and its workstation, was used. All images were recorded digitally through a CCD camera (Gatan MSC791).

\subsection{Small angle $X$-ray scattering}

The SAXS data were recorded with a Kratky compact smallangle system equipped with a position sensitive detector (OED $50 \mathrm{M}$ from Mbraun, Austria) containing 1024 channels of width $53.0 \mu \mathrm{m}$. A monochromator with a $10-\mu \mathrm{m}$-thick nickel filter was used to select the $\mathrm{Cu} K \alpha$ radiation $(\lambda=1.542 \AA)$ provided by the generator, and a $1.5-\mathrm{mm} \mathrm{W}$ filter was used to protect the detector from the primary beam.

The generator (Seifert ID-300 X-ray) was operating at $50 \mathrm{kV}$ and $40 \mathrm{~mA}$. After centrifuging the samples, the precipitate was separated from the supernatant and enclosed in a stainless steel sample holder for solids with mica windows. The distance between the sample and detector was $277 \mathrm{~mm}$. The diffraction patterns were recorded at 25 and $37^{\circ} \mathrm{C}$. The temperature was maintained constant within $0.1^{\circ} \mathrm{C}$ by a Peltier element. The optics and the sample cell were both held under vacuum to minimize the scatter from air.

\section{Results}

\subsection{Characterization of the catanionic vesicle systems}

In previous work we have reported on the phase behavior and aging characteristics of a number of amino-acid-based catanionic mixtures [30], having found that, in analogy with several other mixed cationic-anionic amphiphile systems, it presents a spontaneous formation of vesicles, which have a long-term stability. The reasoning behind the use of an amino-acid-based single-tail amphiphile lays on the fact that ALA presents cell viability percentages above $90 \%$ (results to be published); ALA has also been reported as being biodegradable [18].

The ALA/SCS vesicle mixture used has an ALA to SCS molar charge ratio of 2.9 at $0.5 \mathrm{wt} \%$ in water. The ALA/SOS vesicle mixture used corresponded to a $1 \mathrm{wt} \%$ mixture at a molar charge ratio of 1.7. Both samples presented a bluish look, characteristic of the presence of vesicles in solution [10,31].

In Fig. 2 cryomicrographs of the vesicle solution of ALA/ SOS are represented. Unilamellar and multilamellar vesicles were observed throughout the images taken; a clear perception
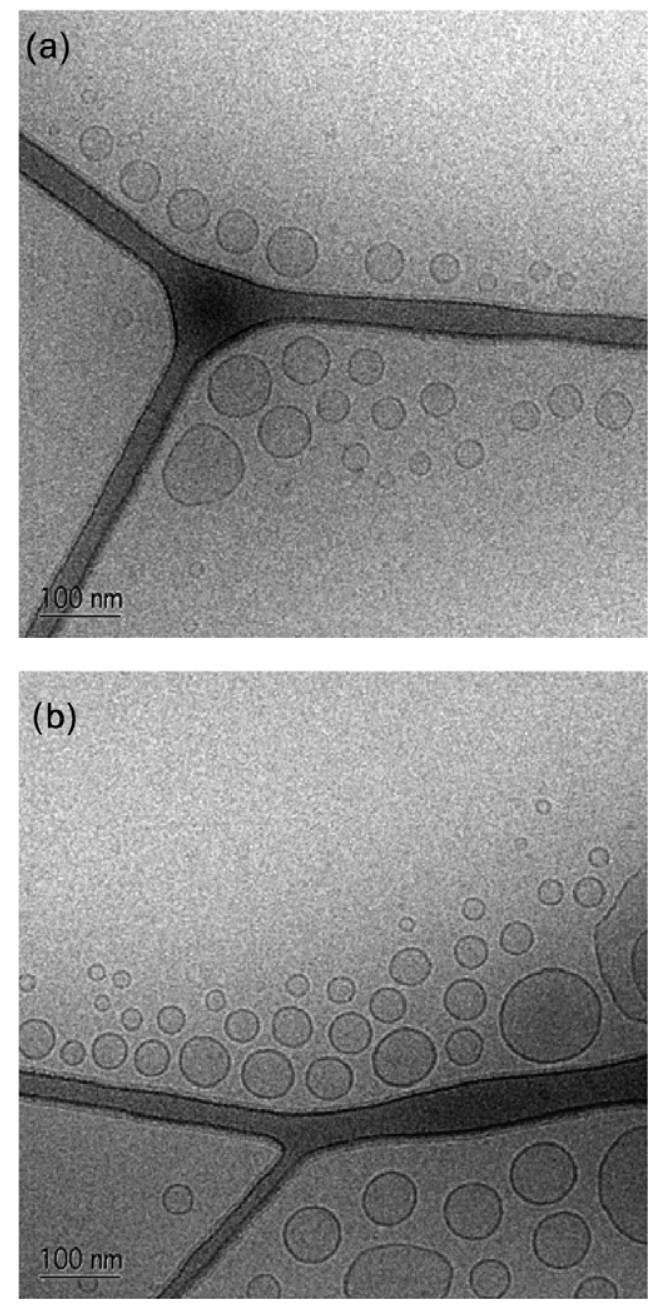

Fig. 2. Cryo-TEM images of the system ALA/SOS/W, 1 wt $\%$, at an ALA/SOS molar charge ratio of 1.7. Unilamellar and multilamellar vesicles were imaged.

of the polydispersity of the vesicles is obtained by looking at all images.

Cryomicrographs for the ALA/SCS vesicle system are exemplified by Fig. 3. As for the previous system, both unilamellar and multilamellar vesicles were observed; the vesicles also show some degree of polydispersity, presenting a tendency for larger vesicles than with SOS as the anionic surfactant.

In order to have a clearer assessment of the degree of polydispersity of these vesicle systems, we monitored a considerable number of vesicles within the cryomicrographs taken. By measuring the diameter of each vesicle we were able to represent the size distribution for the two systems, see Fig. 4. For the SOS-based system, a population of 396 vesicles was analyzed; the number-average radius was determined giving a value of $35.5 \mathrm{~nm}$. For the SCS-based system, a population of 426 vesicles was measured giving a number-average radius of $80.9 \mathrm{~nm}$. Furthermore, by comparing the size distributions in Fig. 4 we can conclude that the SOS-based vesicle system has a lower degree of polydispersity and presents smaller average vesicle sizes. 

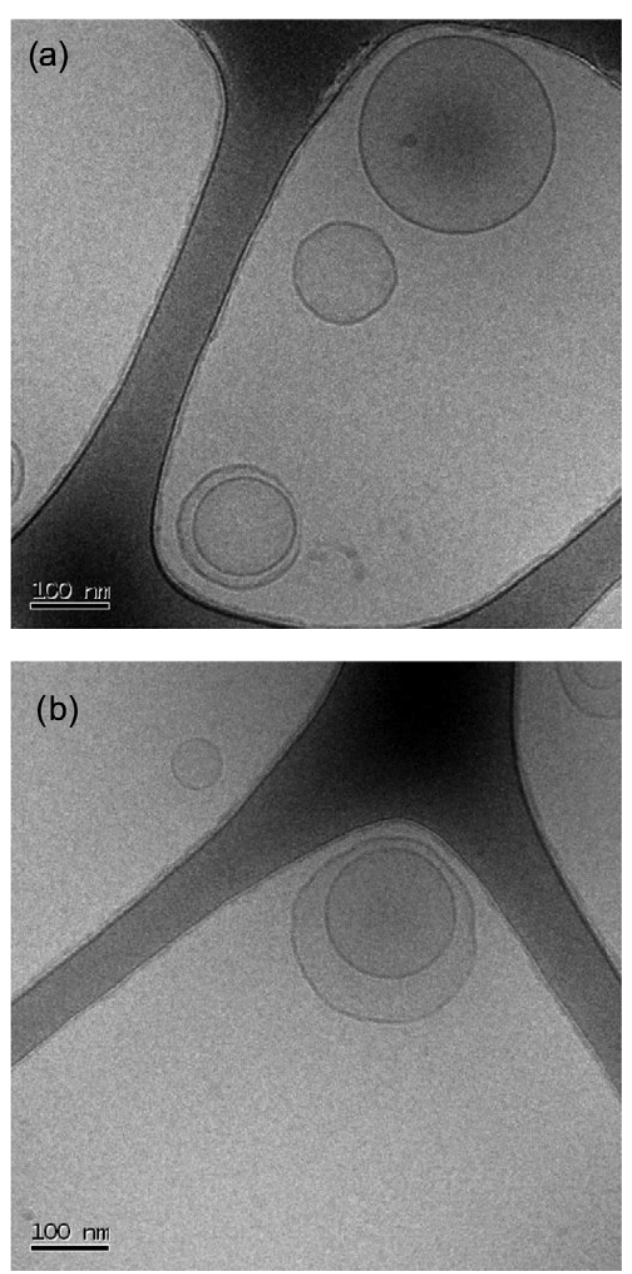

Fig. 3. Cryo-TEM images of the system ALA/SCS/W, $0.5 \mathrm{wt} \%$, at an ALA/SCS molar charge ratio of 2.9. Unilamellar and multilamellar vesicles can be seen throughout the sample.

\subsection{Interaction between DNA and the biocompatible catanionic vesicles}

DNA was added to the vesicle solutions at different ratios of DNA to vesicle effective net charge concentration, $R=$ $[\mathrm{DNA}] /\left[\mathrm{S}^{+}\right]_{\mathrm{eff}}$, where $\left[\mathrm{S}^{+}\right]_{\mathrm{eff}}=[\mathrm{ALA}]-[\mathrm{SOS}$ or SCS $]$; all concentrations are in moles per charge. We are interested in the characterization both of the supernatant and of the precipitated complexes. For the precipitated complexes we explore how the inner microstructure changes with $R$. A correlation between the precipitated complexes and the complexes remaining in the supernatant will be presented.

\subsection{Qualitative inspection of colloidal phase separation}

All mixtures were prepared by adding DNA to the vesicle solutions at different charge ratios, $R$, between DNA and vesicles. Simply by eye inspection no differences in phase separation could be observed between the two systems. Phase separation occurred for all samples, and increased with $R$ until the isoelectric point $(R=1)$, from which point on partial redissolution of the precipitate starts to take place. For $R$ values
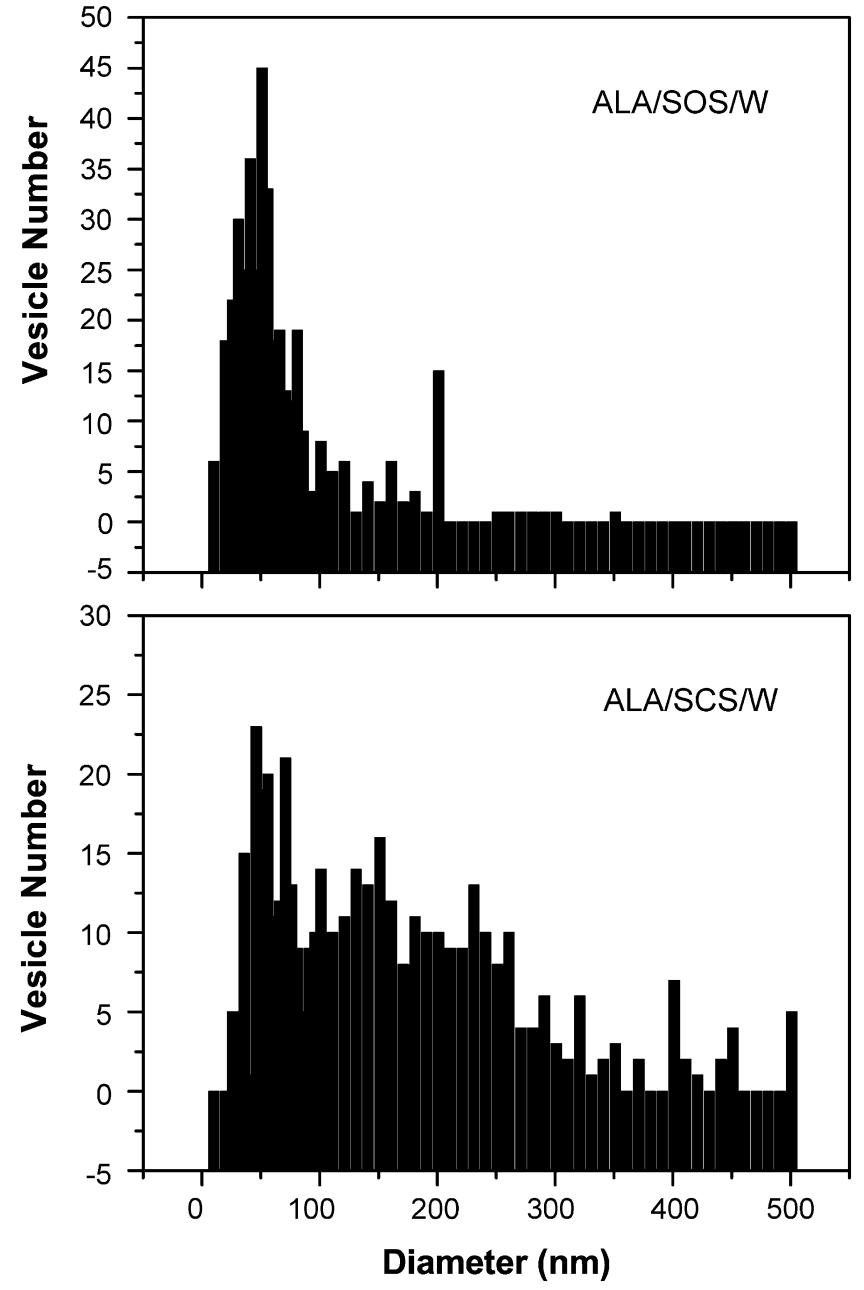

Fig. 4. Vesicle size distribution histograms obtained from cryo-TEM images taken for the vesicle systems ALA/SOS/W and ALA/SCS/W, as depicted in the figure. A higher degree of polydispersity was observed for the system ALA/SCS/W. A population of 396 particles was analyzed for the sample containing SOS and the number-average radius obtained was $35.5 \mathrm{~nm}$. A population of 426 particles was analyzed for the system containing SCS and the number-average radius obtained was $80.9 \mathrm{~nm}$.

below 0.8 , the supernatant presented a turbid bluish look; within $0.8<R<1.2$, a clear solution could be visualized, while for higher $R$ values it became turbid again, but with a more transparent appearance than for lower $R$ values.

\subsection{Microstructure investigation of the concentrated phase}

In Fig. 5 the SAXS spectra for the ALA/SOS/DNA precipitate of samples with $R=0.8,1.0,1.2$ and 1.5, as depicted in the image, can be seen. Due to the redissolution effect mentioned above it was difficult to obtain sufficient amount of precipitate to perform SAXS experiments for higher $R$ values than 1.5. For all samples studied a short-range amphiphile lamellar structure (with $q$ values in the ratios 1:2:3), and with an additional 1D DNA arrangement within these stacks, has been deduced. A repeat distance of $4.7 \mathrm{~nm}$ for the lamellar structure has been calculated for all samples. Correlating this value with the repeat distance of $4.7 \mathrm{~nm}$ obtained from cryo-TEM (see below) for the 


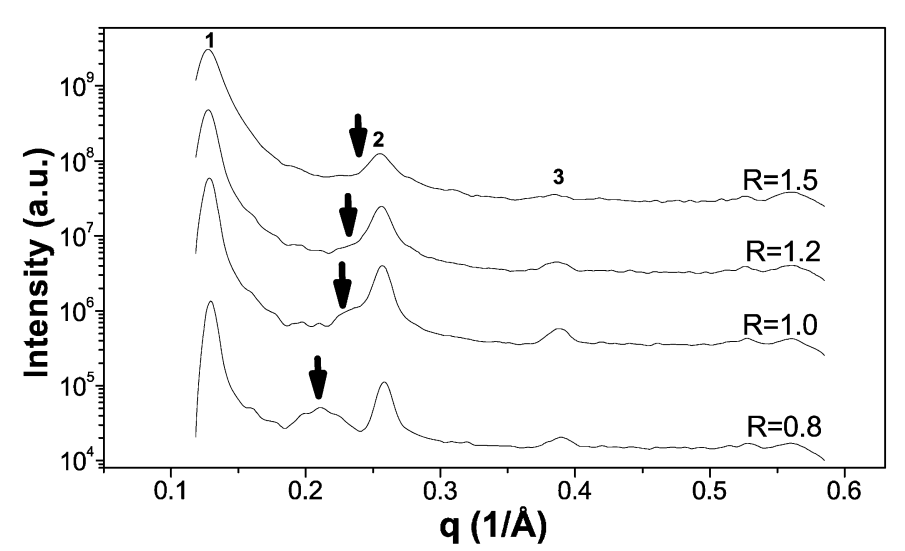

Fig. 5. SAXS diffractograms of precipitated complexes of DNA and SOS-based catanionic vesicles at different DNA to effective cationic amphiphile charge ratios, $R(0.8,1.0,1.2,1.5)$, as depicted in the figure. The arrows indicate the location of the DNA peak. Measurements were performed at both $T=25$ and $37^{\circ} \mathrm{C}$ showing the same results. Here we show the ones performed at $37^{\circ} \mathrm{C}$.

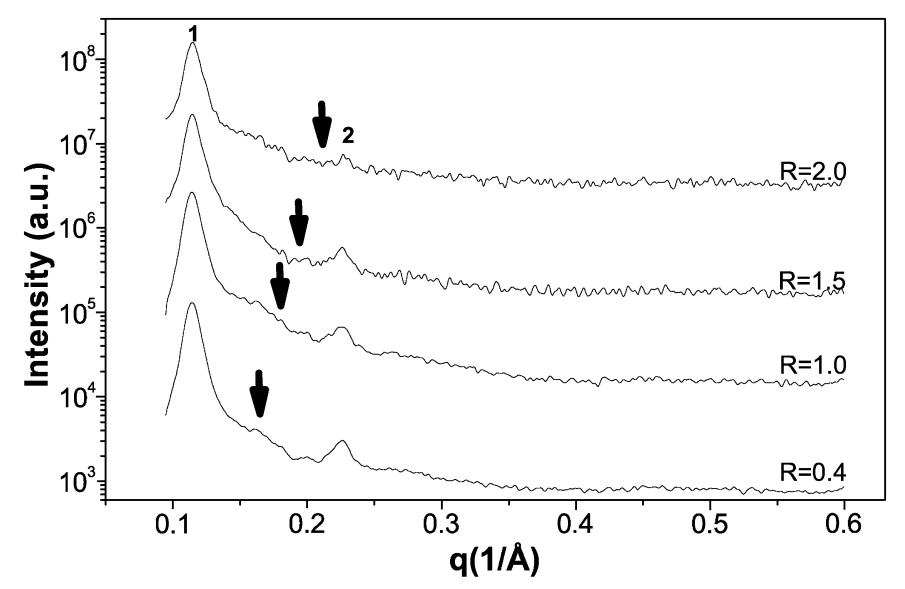

Fig. 6. SAXS diffractograms of precipitated complexes of DNA and SCS-based catanionic vesicles at different DNA to effective cationic amphiphile charge ratios, $R(0.4,1.0,1.5,2.0)$, as shown in the figure. The arrows guide the eye through the DNA peak displacement. Measurements were performed at both $T=25$ and $37^{\circ} \mathrm{C}$ showing the same results. Here we illustrate the ones performed at $37^{\circ} \mathrm{C}$.

sample with $R=1.0$ (Fig. 9b), one can see a good agreement. The additional organization related to the DNA-DNA correlation is indicated by the arrows in the spectra. For $R=0.8$ we can reliably determine the DNA-DNA spacing as being $28 \mathrm{~nm}$. However, from $R=1.0$ and upward, the DNA-DNA spacing peak starts to interfere with the second diffraction peak of the amphiphile organization, with a partial overlap, making it less defined and broader; this makes it difficult to determine the DNA-DNA spacing. Nevertheless, we can safely state that there are higher $q$ values as the amount of DNA increases, indicating that the DNA molecules, as expected, approach each other.

In Fig. 6 the SAXS spectra for the ALA/SCS/DNA precipitate of samples with $R=0.4,1.0,1.5$ and 2.0, as depicted in the image, can be seen. For all samples studied a short-range amphiphile lamellar structure (with $q$ values in the ratios 1:2) with an additional 1D DNA arrangement within these stacks has been deduced. A repeat distance of $5.8 \mathrm{~nm}$ for the lamellar

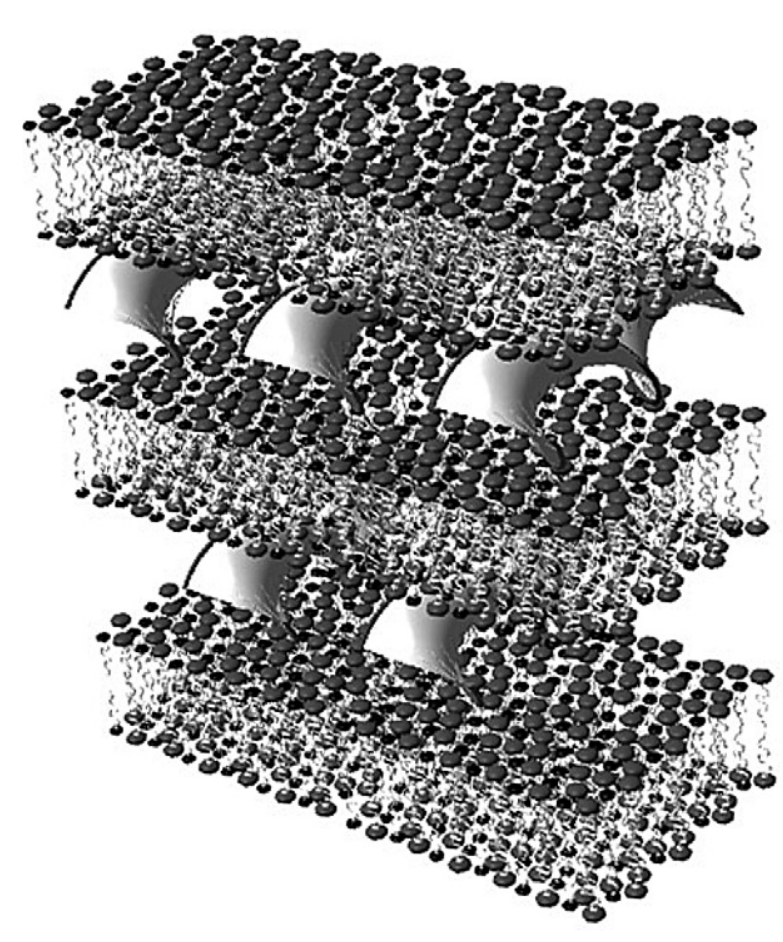

Fig. 7. Schematic representation of the microstructure of the catanionic vesicle-DNA complexes obtained from the SAXS measurements. The DNA molecules are represented by helices on this molecular scale. The anionic (smaller dark heads) and cationic (larger gray heads) amphiphiles comprising the membrane are represented by a globular head with a tail.

structure has been calculated for all samples. Correlating this value with the repeat distance of $6 \mathrm{~nm}$ obtained from cryo-TEM (see below) for the sample with $R=1.0$ (Fig. 12b), one can see a good agreement. The additional organization related to the DNA-DNA correlation is indicated by the arrows in the spectra. We can see that there exists a broad DNA-DNA peak for $R=0.4$ and 1.0. However, for $R=1.5$ and 2.0 the DNA-DNA spacing peak is not as clear. Nevertheless, we believe it presents the same behavior as for the ALA/SOS/DNA spectra- a displacement of the DNA-DNA spacing peak to higher $q$ values with increasing $R$.

A schematic representation of the lamellar phase found for both systems, where the DNA molecules are arranged within the lamellar stacks, is represented in Fig. 7.

\subsection{Observations by cryo-TEM related to the solution complexes}

Cryo-TEM was applied to investigate the size, morphology and inner structure of the complexes formed when oppositely charged vesicles interact with DNA.

From Figs. 8-10 we provide cryo-TEM micrographs, which are representative of the structures found in the studied ALA/ SOS/DNA samples, for different $R$ values.

For the sample with $R=0.4$ (Figs. $8 \mathrm{a}-8 \mathrm{c}$ ), as can be seen from Table 1 , only a fraction of 0.07 of unperturbed vesicles was found; the largest population, with a fraction of 0.80 , being the smaller complexes (total complex area $c<300 \times 300 \mathrm{~nm}^{2}$ ); some larger complexes start to be seen. The few vesicles visual- 

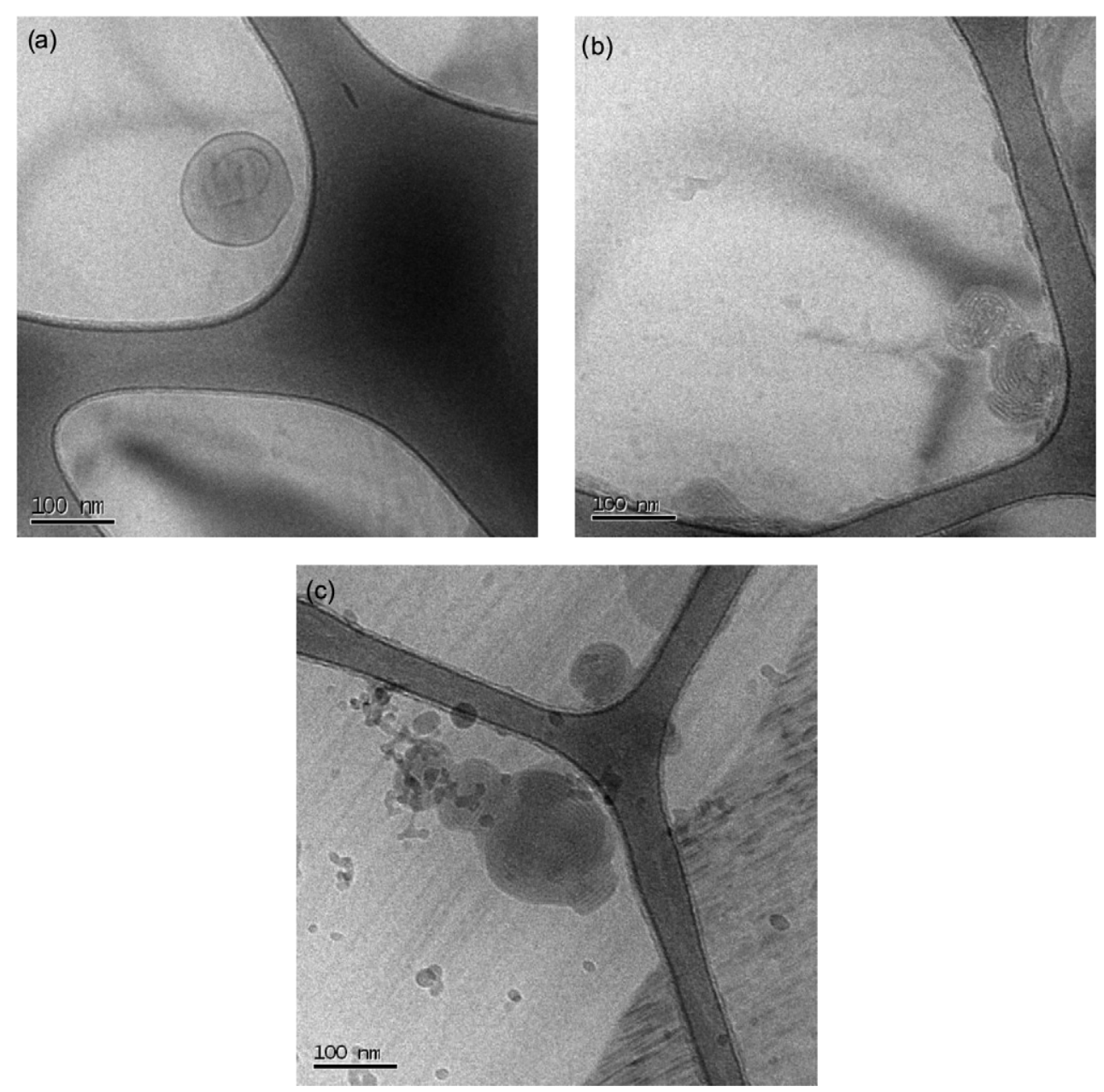

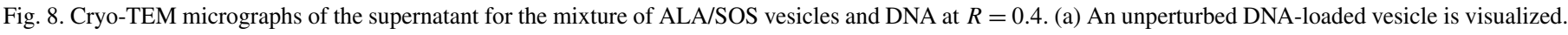
(b, c) DNA-loaded complexes are visualized.

Table 1

Observed fractions of aggregates of different sizes as visualized by cryo-TEM for both systems at different $R$ values, $R=[\mathrm{DNA}] /\left[\mathrm{S}^{+}\right]_{\text {eff }}$

\begin{tabular}{lllll}
\hline System & $R$ & $x_{c<300 \times 300 \mathrm{~nm}^{2}}$ & $x_{c>300 \times 300 \mathrm{~nm}^{2}}$ & $x_{v}$ \\
\hline ALA/SOS/DNA & 0.4 & 0.80 & 0.13 & 0.07 \\
& 1.0 & 0.24 & 0.76 & 0 \\
& 1.5 & 0.52 & 0.48 & 0 \\
ALA/SCS/DNA & 0.4 & 0.19 & 0.04 & 0.77 \\
& 1.0 & 0.66 & 0.34 & 0 \\
& 1.5 & 0.93 & 0.07 & 0 \\
\hline
\end{tabular}

A separation between the fraction of complexes with apparent area size smaller or larger than $300 \times 300 \mathrm{~nm}^{2}$, respectively $x_{c<300 \times 300 \mathrm{~nm}^{2}}$ and

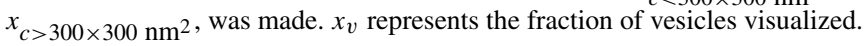

ized seem to be DNA-rich since they were beam sensitive. If we allow the electron beam to irradiate the sample for a little less than one minute the vesicle structures begin to burn and this has been related before to the presence of DNA [32].

For the sample with $R=1.0$ (Figs. 9a-9d) aggregation is at its maximum with the fraction of larger complexes reaching a value of 0.76 . A reorganization of the inner bilayers through merging is evident throughout the cryomicrographs. No vesicles were visualized. An expansion of an area in Fig. 9a, where perfectly parallel bilayers can be visualized, is represented as an inset of the same image. In Fig. 9b, the electron density profile of this inset is also represented; perfectly arranged and equally separated minima give us a bilayer distance of $4.7 \mathrm{~nm}$.

Above the charge equivalence, for $R=1.5$ (Figs. 10a and $10 \mathrm{~b})$, the system has an excess of negative charges giving rise to a swelling effect due to electrostatic repulsion and the fraction of larger aggregates decreases, while the fraction of smaller aggregates increases.

Representative cryomicrographs of the system ALA/SCS/ DNA, at different $R$ values, are illustrated in Figs. 11-13.

For the sample with $R=0.4$ (Figs. 11a-11c) the largest population of unperturbed vesicles was found, with a fraction of 0.77 , as can be seen from Table 1 . By performing the beam irradiation test mentioned above in regions were the ice was vitrified, we deduce that they are loaded with DNA. Some larger complexes start to be seen (0.04), the smaller ones being more frequent $(0.19)$.

At $R=1.0$ (Figs. 12a-12d) no vesicles were visualized; the fraction of aggregated complexes increases, and we obtained a fraction of large complexes $\left(c>300 \times 300 \mathrm{~nm}^{2}\right)$ of 0.34 ; the larger population is the smaller complexes $\left(c<300 \times 300 \mathrm{~nm}^{2}\right)$ with a fraction of 0.66 . As for the SOS-based system, at the 

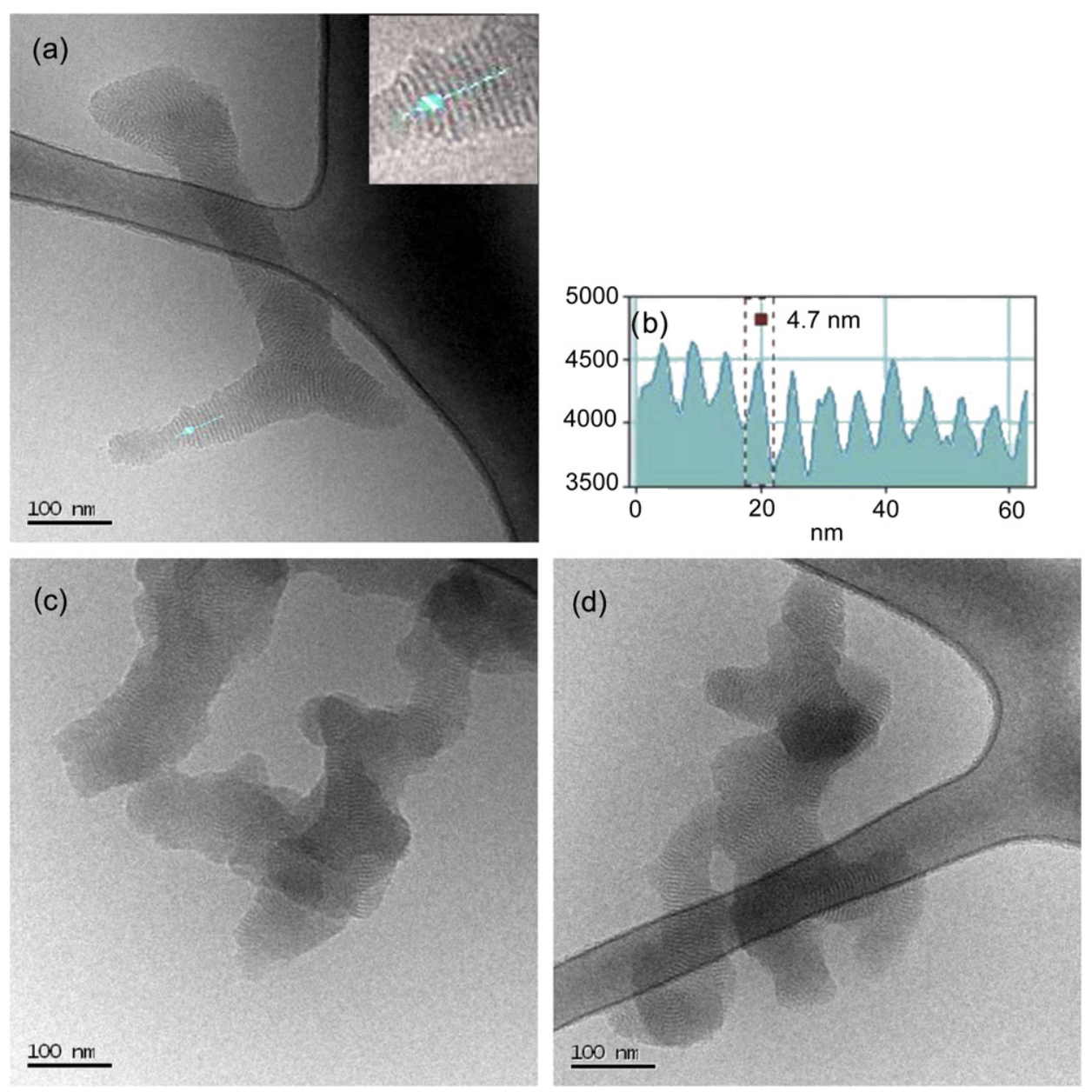

Fig. 9. Cryo-TEM micrographs of the supernatant for the mixture of ALA/SOS vesicles and DNA at $R=1.0$ (a, c, d). A magnified detail of (a) can be seen in the inset of this image; the electron density of the presented line is shown in (b), where a spacing periodicity of $4.7 \mathrm{~nm}$ is shown for the multilamellar structures formed. $T=25^{\circ} \mathrm{C}$.

electroneutral point $(R=1)$, reorganization of the inner bilayers through merging is perceived throughout the images taken. In the inset of Fig. 12a, an expansion of the image, where perfectly parallel bilayers can be visualized, is represented. Its electron density profile gives a repeat distance of $6 \mathrm{~nm}$, as depicted in Fig. 12b.

Above the charge equivalence, for $R=1.5$ (Figs. 13a-13c), due to an excess of negative charges, fragmentation of the aggregates is inferred from the large increase in the fraction of

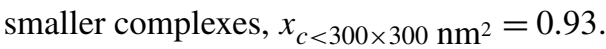

As an overview of the cryo-TEM observations we can make the following comments: initially, for samples with lower DNA content, we see that small globular complexes (Figs. 8 and 11) are more common, and their inner structure is lamellar; at this point, these individual globular complexes can start aggregating leading to a morphologically different complex (Fig. 11b); by adding more DNA the degree of aggregation increases; at the isoelectric point, $R=1$, the individual globular complexes seem to have merged into each other by rearranging and reorienting their inner bilayers; as a result we no longer see the individual complexes that form the particle (Figs. 9 and 12); above the isoelectric point the complexes seem to gradually lose their internal organization and fragment into smaller complexes (Figs. 10 and 13).

An important aspect emerges from the above overview-the ability to manipulate and control the internal structure, the morphology and the size of the complexes formed by changing only one parameter: the charge ratio between DNA and the catanionic vesicles. Furthermore, by changing a component within the mixture (SOS or SCS), some features also change, the most preeminent being the spacing between the lamellas, which increases with the alkyl chain length of the amphiphile.

\section{Discussion}

\subsection{Microstructure of the DNA/vesicle complexes}

\subsubsection{Concentrated phase}

As mentioned above, when performing SAXS measurements on the precipitated complexes at different $R$ values we obtained spectra characteristic of a lamellar structure with an additional diffraction peak from the DNA arrangement within the lamellar stacks. The described structure presents a characteristic repeat distance and a DNA-DNA spacing which de- 

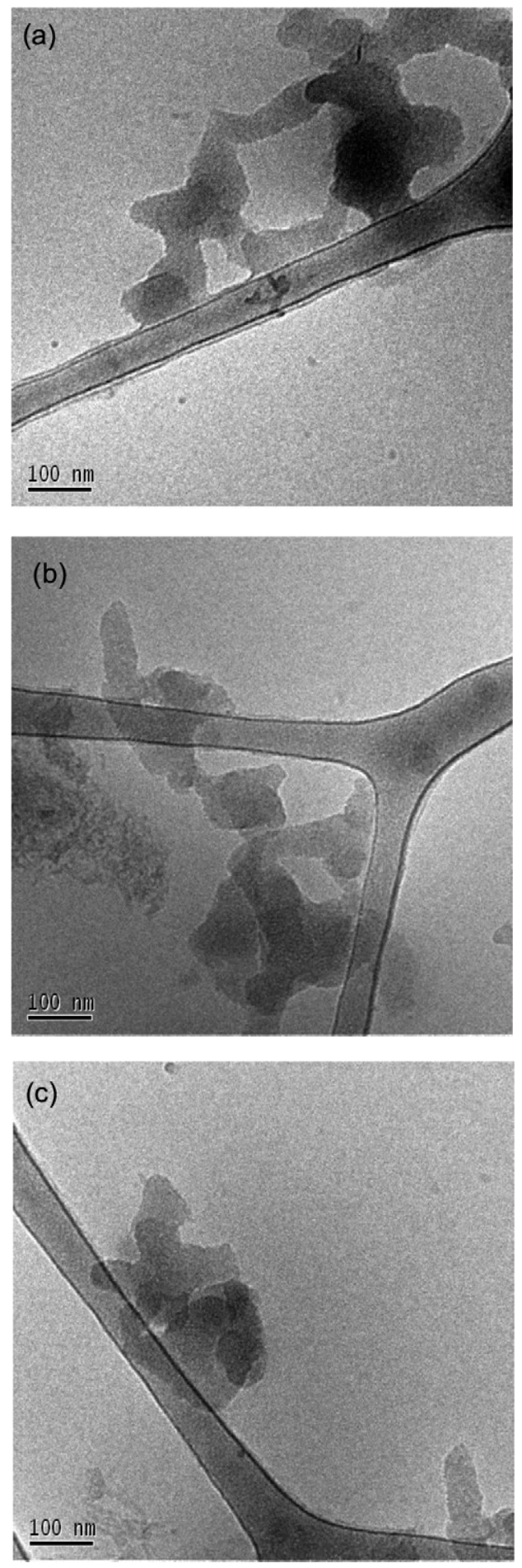

Fig. 10. Cryo-TEM micrographs of the supernatant for the mixture of ALA/SOS vesicles and DNA at $R=1.5 . T=25^{\circ} \mathrm{C}$.

creases as more DNA is added (better seen for the SOS system). This can be interpreted in terms of an increasing introduction of DNA into the lamellar phase. This is compensated by a release of the anionic amphiphile; the bilayers, therefore, become increasingly positively charged and compensate for the increased DNA concentration in the water layers of the lamellar phase. The formation of this kind of structure has previously been reported in the literature for lipidic and amphiphilic-based sys-
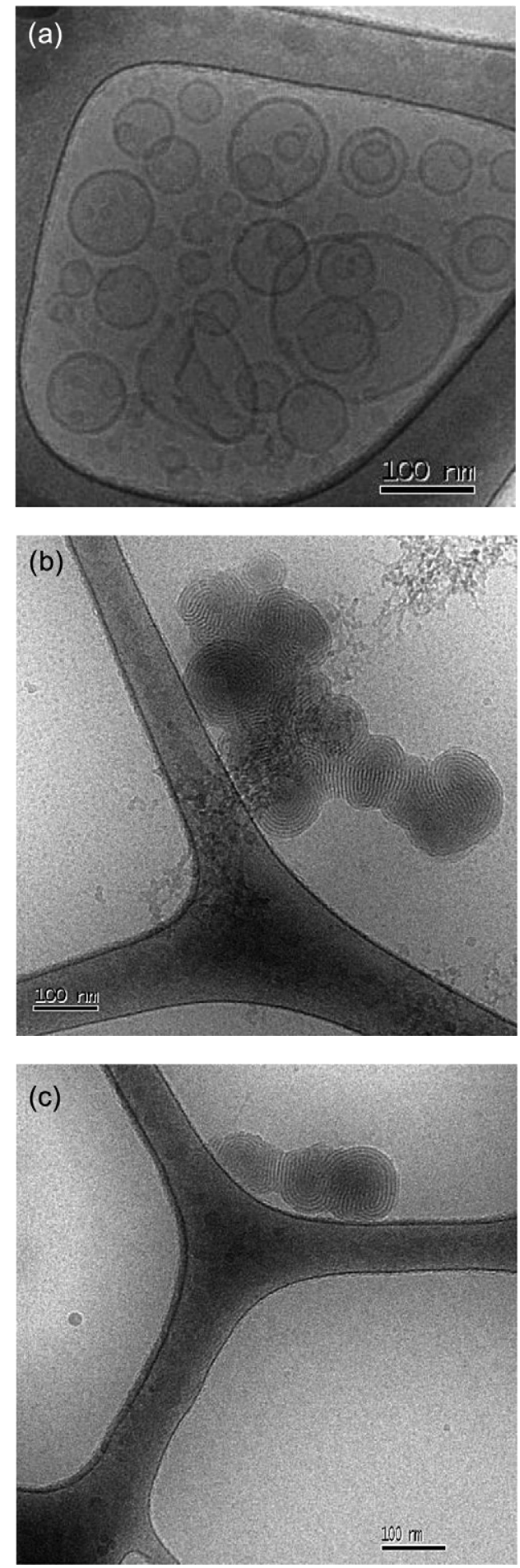

Fig. 11. Cryo-TEM micrographs of the supernatant for the mixture of ALA/SCS vesicles and DNA at $R=0.4$. (a) Unperturbed DNA-loaded vesicles are visualized. (b, c) Perturbed DNA-loaded complexes are visualized.

tems and DNA [14,16,33-35]. Furthermore, we believe that the fact that the DNA-DNA spacing decreases for $R$ values above 1 is characteristic for catanionic-based systems, and that it represents an advantage from a transfection point of view [36] since it allows further compaction of DNA compared with liposomebased systems, where, after reaching the charge stoichiometry between cationic lipid and DNA, further addition of DNA does 

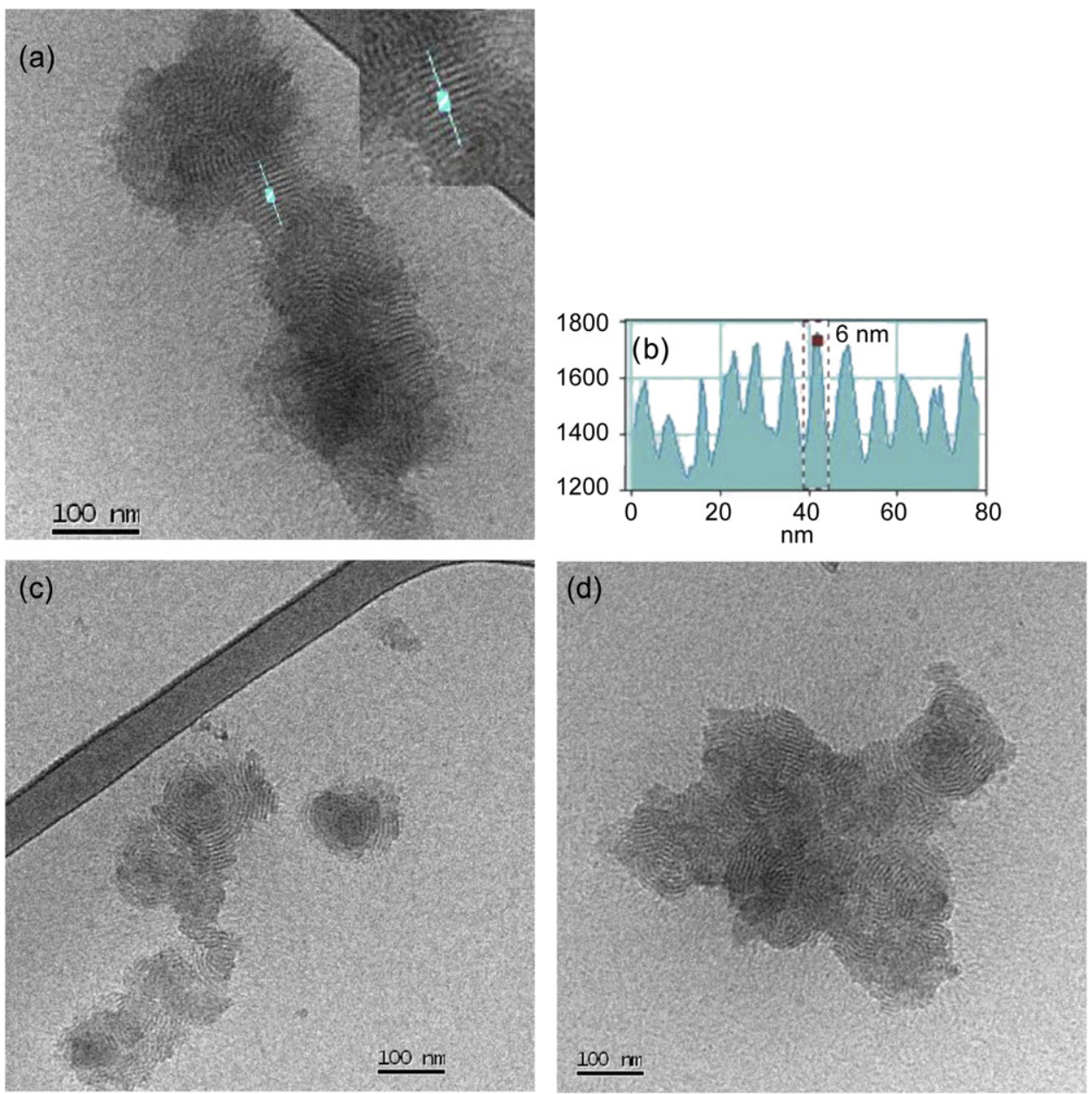

Fig. 12. Cryo-TEM micrographs of the supernatant for the mixture of ALA/SCS vesicles and DNA at $R=1.0$ (a, c, d). A magnified detail of (a) can be seen in the inset of this image; the electron density of the presented line is shown in (b), where a spacing periodicity of 6.0 nm is shown for the multilamellar structures formed. $T=25^{\circ} \mathrm{C}$.

not result in a decrease in the DNA-DNA spacing within the complex [37].

As a rough estimate, we can calculate the repeat distances for the systems studied and compare with the experimental results obtained. For that we need to consider the maximum length of a fully extended alkyl chain to be given in nanometers $(\mathrm{nm})$ by the equation $l=0.15+0.127 n_{\mathrm{c}}$, where $n_{\mathrm{c}}$ is the number of carbon atoms in the chain [38]. From the literature we also know that in the liquid state the membrane thickness is approximately $1.6 l_{\max }$. Thus, for the SOS-based system, since we have a mixture of 12 and 8 carbon alkyl chains, we would have $l_{\mathrm{ALA}}=$ $1.67 \mathrm{~nm}$ and $l_{\mathrm{SOS}}=1.17 \mathrm{~nm}$; as the bilayer membrane is constituted by both amphiphiles we would have an intermediate membrane thickness of $d_{\mathrm{mem}}=1.6\left(l_{\mathrm{ALA}}+l_{\mathrm{SOS}}\right) / 2=2.27 \mathrm{~nm}$. For the SCS-based system, we get $l_{\mathrm{ALA}}=1.67 \mathrm{~nm}$ and $l_{\mathrm{SCS}}=$ $2.18 \mathrm{~nm}$ and $d_{\mathrm{mem}}=1.6\left(l_{\mathrm{ALA}}+l_{\mathrm{SCS}}\right) / 2=2.91 \mathrm{~nm}$. Taking into account the presence of DNA and considering that a DNA molecule with one hydration shell has a diameter of ca. $2.5 \mathrm{~nm}$, we would get a repeat distance of approximately $4.77 \mathrm{~nm}$ for the SOS-based system and of $5.41 \mathrm{~nm}$ for the SCS-based one. For the SOS-based system the calculated value of $4.77 \mathrm{~nm}$ is quite close to the one obtained experimentally. However, for the SCS-based system, we expected that SCS should have a larger contribution to the bilayer thickness; actually, if we try to fit the bilayer thickness formula $d_{\mathrm{mem}}=1.6\left(\left(1-x_{\mathrm{SCS}}\right) l_{\mathrm{ALA}}+\right.$ $\left.x_{\mathrm{SCS}} l_{\mathrm{SCS}}\right), x_{\mathrm{SCS}}$ being the fraction of SCS molecules contributing to the bilayer thickness, to the estimated spacing of $3.3 \mathrm{~nm}$ $\left(d_{\text {mem }}=D_{\mathrm{SAXS}}-d_{\mathrm{DNA}}=5.8-2.5=3.3 \mathrm{~nm}\right)$, we get a value of $x_{\mathrm{SCS}}$ equal to 0.77 , thus giving an indication that for this systems SCS gives a larger contribution to the bilayer thickness. These results indicate that both amphiphiles are contributing to the final bilayer thickness, instead of the expected result that the longer alkyl chain amphiphile alone controls it.

\subsubsection{Solution complexes}

Cryo-TEM images together with the calculated fractions of the different complexes, shown in Table 1, give a good insight into the association behavior in the supernatant solution. As DNA is added to the vesicle solution, small globular complexes start to form at low concentrations; at this point vesicles are still visualized in solution, with the difference of containing DNA; a limited degree of aggregation of the globular complexes is detected; increasing the DNA content leads to an increasing degree of complex aggregation; larger clusters are formed as noted from the maximum in the fraction of larger ag- 

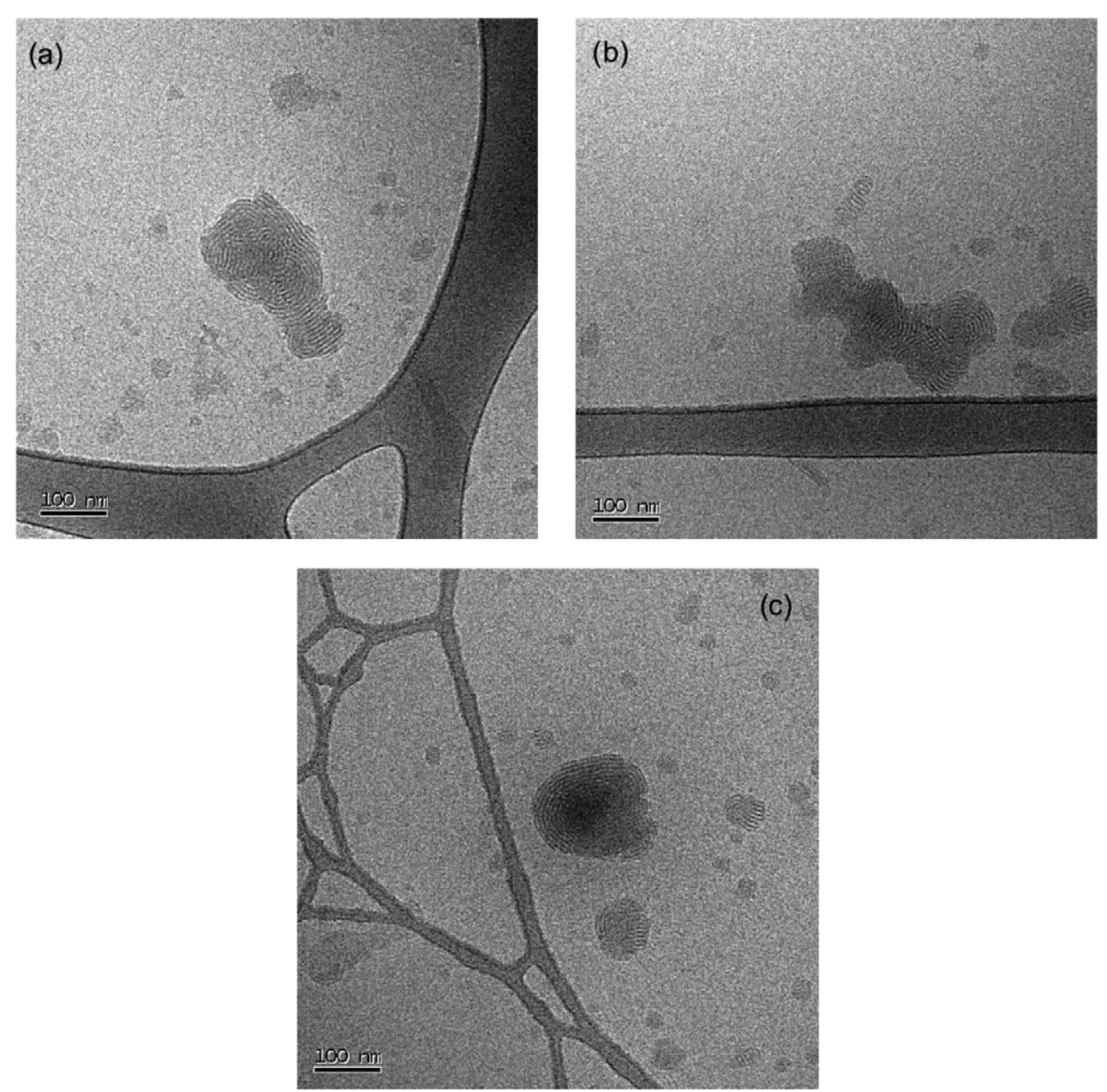

Fig. 13. Cryo-TEM micrographs of the supernatant for the mixture of ALA/SCS vesicles and DNA at $R=1.5$. (a-c) Small complexes ( $\ll 100 \mathrm{~nm}$ ) dispersed within the sample are visualized. $T=25^{\circ} \mathrm{C}$.

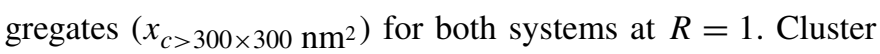
formation at electroneutrality has been previously seen in the studies of polyelectrolyte-oppositely charged amphiphile systems, both experimentally [14,37,39-41] as well as in Monte Carlo simulations [42]. Above the isoelectric point, the clusters start to become smaller and the complexes begin drifting apart from each other, due to electrostatic repulsions generated by the excess of negative charges in the structures. This decrease in aggregation has been reported previously for DNA-cationic liposome complexes [37], and has been related with charge reversal of the complexes due to excess of DNA [37]. In a study on DNA in the presence of oppositely charged catanionic vesicles, it was demonstrated that excess of DNA did not lead to the coexistence of DNA-vesicle complexes and DNA [37], but most probably to the inclusion of DNA in excess in the complexes and, therefore, to a coexistence of complexes and anionic amphiphile micelles expelled from the bilayers [16].

\subsection{Remarks on the phase behavior}

The interaction between oppositely charged amphiphiles and DNA is strongly attractive. No qualitative differences in phase behavior were observed between the ALA/SOS/DNA and ALA/SCS/DNA systems. Precipitation was observed for all mixing ratios, which correlates well with a strong association. As the concentration of DNA increases in the vesicle solution, the amount of precipitate increases till we reach charge neutrality $(R=1)$; from this point on, as more polyelectrolyte is added into solution, redissolution of the precipitate starts to be noticed. These features are commonly associated with polyelectrolyte-oppositely charged amphiphile systems [43-45], as well as oppositely charged mixed amphiphilepolyelectrolyte systems [41]. It has been shown by means of Monte Carlo simulations, that at charge equivalence, the system becomes unstable and phase separates [42] and that further addition of polyelectrolyte resulted in a single and stable macroion-polyelectrolyte solution due to a redissolution effect [42]. This has been experimentally shown by Carnali [45], where complete redissolution of the precipitate was observed on the addition of excess polyelectrolyte; by adding even more polyelectrolyte, another phase separation occurred, this time of the segregative type [45]. In contrast to this, studies on DNAoppositely charged catanionic vesicle systems showed that excess DNA does not induce complete redissolution of the precipitate $[14,16]$, as we also observed for the two systems considered in the present work. Thus, we can assume that the introduction of an amino-acid-based amphiphile in the catanionic system does not give rise to qualitative differences in the phase be- 
havior of mixed DNA-amphiphile systems as compared to classical amphiphiles [14]. The main advantage of the amino-acidbased systems presented here is the possibility of using them in real biological applications, having as a tool the physical chemistry knowledge obtained from studying more classical systems.

\section{Conclusions}

The interaction between positively charged amino-acidbased catanionic vesicles is, as expected, strongly associative. Mixing DNA and vesicles in solution leads to phase separation for all mixing ratios between DNA and the catanionic mixtures studied; above the isoelectric point, partial redissolution of the complexes is observed. The inner structure of the precipitate corresponds to organization in bilayers of both amphiphiles, while DNA occupies the interbilayer gap. By substituting SOS for SCS, an increase in the repeat distance of the complexes, from 4.7 to $5.8 \mathrm{~nm}$, is denoted; SCS seems to have a higher contribution to the final bilayer thickness within the complexes. DNA molecules located within these lamellar stacks present a characteristic spacing which decreases on further DNA addition. In the supernatant, as the DNA content increases, the individual complexes start to aggregate forming large clusters; above the isoelectric point, due to an excess of negative charges, the clusters disintegrate and the complexes seem to fragment, decreasing their sizes.

\section{Acknowledgments}

We are grateful to Gunnel Karlsson for aid in cryo-TEM instrumentation. We would like to thank Maria Rosa Infante and María del Carmen Morán for the generous synthesis and offer of the amino-acid-based surfactant (ALA). This work was supported by grants from the Fundação para a Ciência e Tecnologia (FCT, Project POCTI SFRH/BD/8357/2002, FEDER-POCTI/ QUI/58689/2004 and POCTI/QUI/45344/02), the Swedish Research Council (VR) and a grant from an EU Research Training Network, CIPSNAC (Contract MRTN-CT-2003-504932).

\section{References}

[1] P.L. Felgner, T.R. Gadek, M. Holm, R. Roman, H.W. Chan, M. Wenz, J.P. Northrop, G.M. Ringold, M. Danielsen, Proc. Natl. Acad. Sci. USA 84 (21) (1987) 7413-7417.

[2] A. El-Aneed, J. Controlled Release 94 (2004) 1-14.

[3] H.F. Willard, Science 290 (5495) (2000) 1308-1309.

[4] S.E. Raper, N. Chirmule, F.S. Lee, N.A. Wivel, A. Bagg, G.P. Gao, J.M. Wilson, M.L. Batshaw, Mol. Gen. Metabol. 80 (1-2) (2003) 148-158.

[5] S. Hacein-Bey-Abina, C. von Kalle, M. Schmidt, F. Le Deist, N. Wulffraat, E. McIntyre, I. Radford, J.L. Villeval, C.C. Fraser, M. Cavazzana-Calvo, A. Fischer, N. Engl. J. Med. 348 (3) (2003) 255-256.

[6] C. Dufes, W.N. Keith, A. Bilsland, I. Proutski, I.F. Uchegbu, A.G. Schatzlein, Cancer Res. 65 (18) (2005) 8079-8084.

[7] A.S. Ulrich, Biosci. Rep. 22 (2) (2002) 129-150.

[8] E.W. Kaler, A.K. Murthy, B.E. Rodriguez, J.A.N. Zasadzinski, Science 245 (4924) (1989) 1371-1374.

[9] E.W. Kaler, K.L. Herrington, A.K. Murthy, J.A.N. Zasadzinski, J. Phys. Chem. 96 (16) (1992) 6698-6707.

[10] M.T. Yatcilla, K.L. Herrington, L.L. Brasher, E.W. Kaler, S. Chiruvolu, J.A. Zasadzinski, J. Phys. Chem. 100 (14) (1996) 5874-5879.
[11] E.F. Marques, O. Regev, A. Khan, M.D. Miguel, B. Lindman, J. Phys. Chem. B 102 (35) (1998) 6746-6758.

[12] E.F. Marques, O. Regev, A. Khan, M.D. Miguel, B. Lindman, J. Phys. Chem. B 103 (39) (1999) 8353-8363.

[13] S.M. Mel'nikov, R. Dias, Y.S. Mel'nikova, E.F. Marques, M.G. Miguel, B. Lindman, FEBS Lett. 453 (1-2) (1999) 113-118.

[14] R.S. Dias, B. Lindman, M.G. Miguel, J. Phys. Chem. B 106 (48) (2002) 12600-12607.

[15] R.S. Dias, B. Lindman, M.G. Miguel, J. Phys. Chem. B 106 (48) (2002) 12608-12612.

[16] M. Rosa, M.C. Morán, M.G. Miguel, B. Lindman, Colloids Surf. A Physicochem. Eng. Aspects, submitted for publication.

[17] K. Wagner, D. Harries, S. May, V. Kahl, J.O. Rädler, A. Ben-Shaul, Langmuir 16 (2) (2000) 303-306.

[18] C. Moran, P. Clapes, F. Comelles, T. Garcia, L. Perez, P. Vinardell, M. Mitjans, M.R. Infante, Langmuir 17 (16) (2001) 5071-5075.

[19] G. Byk, C. Dubertret, V. Escriou, M. Frederic, G. Jaslin, R. Rangara, B. Pitard, J. Crouzet, P. Wils, B. Schwartz, D. Schermann, J. Med. Chem. 41 (2) (1998) 224-235.

[20] J.S. Remy, C. Sirlin, P. Vierling, J.P. Behr, Bioconjugate Chem. 5 (6) (1994) 647-654.

[21] L.L. Brasher, K.L. Herrington, E.W. Kaler, Langmuir 11 (11) (1995) 4267-4277.

[22] M.R. Infante, A. Pinazo, J. Seguer, Colloids Surf. A Physicochem. Eng. Aspects 123 (1997) 49-70.

[23] C. Moran, A. Pinazo, P. Clapes, L. Perez, P. Vinardell, M.R. Infante, Green Chem. 6 (5) (2004) 233-240.

[24] J. Sambrook, E.F. Fritsch, T. Maniatis, Molecular Cloning: A Laboratory Manual, Cold Spring Harbor Laboratory Press, New York, 1989.

[25] W. Saenger, Principles of Nuclei Structure, Springer-Verlag, New York, 1984.

[26] T. Akao, T. Osaki, J. Mitoma, A. Ito, T. Kunitake, Bull. Chem. Soc. Jpn. 64 (12) (1991) 3677-3681.

[27] J.R. Bellare, H.T. Davis, L.E. Scriven, Y. Talmon, J. Electron Microsc. Tech. 10 (1) (1988) 87-111.

[28] Y. Talmon, Ber. Bunsen-Ges. Phys. Chem. Chem. Phys. 100 (3) (1996) 364-372.

[29] M. Adrian, J. Dubochet, J. Lepault, A.W. McDowall, Nature 308 (5954) (1984) 32-36.

[30] M. Rosa, M.R. Infante, M. Miguel, B. Lindman, Langmuir 22 (13) (2006) 5588-5596.

[31] E.F. Marques, Langmuir 16 (11) (2000) 4798-4807.

[32] E.K. Wasan, P. Harvie, K. Edwards, G. Karlsson, M.B. Ballys, Biochim. Biophys. Acta Biomembr. 1461 (1) (1999) 27-46.

[33] J.O. Rädler, I. Koltover, T. Salditt, C.R. Safinya, Science 275 (5301) (1997) 810-814.

[34] B.J. Battersby, R. Grimm, S. Huebner, G. Cevc, Biochim. Biophys. Acta Biomembr. 1372 (2) (1998) 379-383.

[35] Y.S. Mel'nikova, S.M. Mel'nikov, J.E. Löfroth, Biophys. Chem. 81 (2) (1999) 125-141.

[36] S. Audouy, D. Hoekstra, Mol. Membr. Biol. 18 (2) (2001) 129-143.

[37] J.O. Rädler, I. Koltover, A. Jamieson, T. Salditt, C.R. Safinya, Langmuir 14 (15) (1998) 4272-4283.

[38] D.F. Evans, H. Wennerström, The Colloidal Domain-Where Physics, Chemistry, Biology and Technology Meet, second ed., Wiley-VCH, New York, 1999.

[39] J. Gustafsson, G. Arvidson, G. Karlsson, M. Almgren, Biochim. Biophys. Acta Biomembr. 1235 (2) (1995) 305-312.

[40] S. Huebner, B.J. Battersby, R. Grimm, G. Cevc, Biophys. J. 76 (6) (1999) 3158-3166.

[41] E.F. Marques, O. Regev, A. Khan, M.D. Miguel, B. Lindman, Macromolecules 32 (20) (1999) 6626-6637.

[42] M. Skepö, P. Linse, Macromolecules 36 (2) (2003) 508-519.

[43] K. Thalberg, B. Lindman, K. Bergfeldt, Langmuir 7 (1991) 2893-2898.

[44] K. Thalberg, B. Lindman, G. Karlström, J. Phys. Chem. 95 (15) (1991) 6004-6011.

[45] J.O. Carnali, Langmuir 9 (11) (1993) 2933-2941. 